\title{
USO DE NUEVAS HERRAMIENTAS PARA EL CONTROL DE TRIATOMINOS EN DIFERENTES SITUACIONES ENTOMOLÓGICAS EN EL CONTINENTE AMERICANO
}

\begin{abstract}
Alfredo M. Oliveira Filho
Se relatan los resultados obtenidos en el terreno seis meses y dos años posttratamiento con las nuevas herramientas para el control de triatominos desarrolladas con apoyo de la OMS/TDR, o sea, a) pinturas insecticidas, una suspensión emulsionable de lenta liberación conteniendo el $8,3 \%$ de malathion, aplicada por rociamiento; b) potes fumígenos que, después de encendidos, echan bumos que contienen cypermetrina y DDVP; c) cajas sensoras para detección de triatominos. Los ensayos en el terreno fueron realizados en Chile. Honduras y Paraguay contra tres especies principales de vectores de la enfermedad de Chagas - Triatoma infestans, T. dimidiata $y$ Rhodnius prolixus, según el protocolo elaborado por un comité de expertos de la OMS. Los resultados indican que las pinturas insecticidas constituyen un eficaz medio de control de estos vectores en cualquiera de las condiciones estudiadas, sea en el domicilio, sea en el peridomicilio, manteniendo los porcentajes de infestación muy próximos a cero. Comparaciones preliminares de la técnica de cajas sensoras para detección de casas infestadas por triatominos, con la búsqueda hecha por personal entrenado (hora/hombre) indican esta última como la mas apropiada.
\end{abstract}

Palabras-llaves: Insecticidas. Lenta liberación. Triatominos. Enfermedad de Chagas.

El mejoramiento de la vivienda rural o periurbana, asociado con educación sanitaria de la población humana que vive en áreas endémicas para la enfermedad de Chagas, todo esto hecho preferencialmente con intensa participación popular, sigue siendo una recomendación constante de muchos de los organismos mundiales que tratan la salúd publica y el bienestar social ${ }^{14}$. Esto ocurre no sólo porque se puede disminuir significativamente la transmisión del Trypanosoma cruzi por los triatominos vectores, sino también porque mejora la calidad de vida de los habitantes, disminuyendo consecuentemente la prevalencia de otras enfermedades transmisibles. Sin embargo, el tratamiento de la vivienda con insecticidas de acción residual sigue siendo el método preferido por las autoridades sanitarias responsables de las campañas de control de la enfermedad de Chagas en Latinoamérica. Esto se debe a su respuesta rápida en la eliminación de las vinchucas, al costo/casa relativamente bajo y a la facilidad de implantación de un

Núcleo de Pesquisas de Produtos Naturais da Universidade Federal do Rio de Janeiro, Rio de Janeiro, RJ.

Endereço para correspondência: Dr. Alfredo M. Oliveira Filho. CCS/NPPN/UFRJ. Av. Brigadeiro Trompowsky s/n, Bl. H, 21941-590 Rio de Janeiro, RJ, Brasil. Fax 55-21-2703883. Recebido para publicação em 01/12/95. programa para tratamiento de grandes áreas, cuando se compara con los métodos mencionados al principio.

El análisis del control de vectores con insecticidas casi siempre lleva a la consideración de por qué las poblaciones de estos insectos se recuperan después de un intervalo postaplicación, en general de algunos meses. Está claro que estos individuos pueden ser remanentes del tratamiento o reinfestantes de fuera. De todos modos, si el insecticida tuviera suficiente persistencia en los locales tratados, se evitarían tanto la sobrevivencia como la entrada y colonización por nuevos individuos y con esto también la necesidad de nuevos tratamientos costosos y constantes. Por esta razón nuestro laboratorio estuvo estudiando y desarrollando, durante años, formulaciones de lenta liberación que preservan los insecticidas incorporados de la rápida degradación. Uno de estos productos, basados en una matriz de acetato de polivinilo (PVA) y copolímeros conteniendo el organofosforado malathion, ha demostrado gran eficacia en ensayos en el terreno en Brasil, controlando el T. infestans y T. sordida, en el intra o en el peridomicilio, con una sola aplicación, durante casi dos años ${ }^{456}$ y, además, con un costo/eficacia favorable en relación a tratamientos convencionales ${ }^{7}$. 
Oliveira Filho AM. Uso de nuevas herramientas para el control de triatominos en diferentes situaciones entomológicas en el continiente americano. Revista da Sociedade Brasileira de Medicina Tropical 30:41-46, jan-fev, 1997.

En consecuencia de estos resultados obtenidos, en los ensayos realizados en conjunto con el Ministerio de Salud de Brasil, la OMS/TDR propuso la realización de nuevos ensayos en el terreno en varios países, buscando estudiar el uso de pinturas insecticidas (como así pasó a ser conocida la formulación, a pesar de que no es en realidad, una pintura) en el control de triatominos en diferentes situaciones entomológicas en el continente americano. Para esto invitó, del 23 al 26 de Octubre de 1989, en Montevideo, a un grupo alrededor de 40 expertos en control de vectores, técnicos, estadísticos, epidemiólogos, sociólogos y economistas para la elaboración del "Protocolo Estandar para el Ensayo de Nuevas Estrategias de Control de Vectores de la Enfermedad de Chagas".

Los objetivos de este ensayo eran los de evaluar el impacto, el costo-eficacia y la aceptabilidad de dos nuevas herramientas para el control de triatominos en las viviendas y, consecuentemente, sobre los índices de infección por Trypanosoma cruzi en la población humana. Una de las herramientas se constituía de la pintura insecticida con el 8,3\% de malathion en PVA y copolímeros para aplicación a $2 \mathrm{~g}$ de i.a. $/ \mathrm{m}^{2}$; y la otra de potes fumígenos que, encendidos, echaban humos con insectidas (organofosforados y piretroides), desarrollados en el Instituto de Investigaciones Científicas y Técnicas de Buenos Aires ${ }^{14}$. Estas nuevas técnicas serían evaluadas en comparación con aplicaciones tradicionales de insecticidas residuales. Los principales vectores bajo ataque serían los T. infestans, T. dimidiata y Rhodnius prolixus.

Para detectar infestaciones se evaluaria también una versión de la caja de GómezNuñez: los "Sensores María" (cajas de cartón con papel absorbente doblado en su interior) que, fijados a las paredes de la casa, pueden detectar pasivamente la presencia de triatominos. Esto se hace por la recolección directa de los insectos cuando se efectúa la supervisión periódica de las cajas, o por sus señales, tales como heces, huevos o exuvias. Estos resultados serían comparados con los del método de búsqueda hora/hombre para la vigilancia epidemiológica y evaluación de las intervenciones de control.

Cinco países firmaron un acuerdo con la OMS/TDR para la realización del ensayo en su territorio: Argentina, Bolivia, Chile, Honduras y
Paraguay. En este trabajo preliminar se presentaron los datos disponibles, recibidos por la OMS-Ginebra, en los relatos enviados por los investigadores de Chile (D. Julio Valdés y Dr. Víctor Correa), Honduras (Drs.Carlos y Elisabeth Ponce) y Paraguay (Dr $\stackrel{a}{ }$ Sofia Cardozo).

\section{MATERIAL Y MÉTODOS}

La pintura de lenta liberación de insecticida fue producida, bajo encargo y especificación de nuestro laboratorio, por la compañía Selassol de S. Paulo, Brasil, con el nombre de LONG ACTION M10, conteniendo el 8,3\% de malathion. Los embalajes contenían 5 litros del producto para ser mezclados a otros 5 litros de agua, haciendo así una carga de 10 litros, lo suficiente para cubrir $200 \mathrm{~m}^{2}$, resultando en una dosis final de $2 \mathrm{~g}$ de i.a. $/ \mathrm{m}^{2}$. La aplicación única fue hecha con bombas dorsales, manuales de distintas marcas, pero siempre utilizando boquillas Teejet 8002 , a una distancia de $45 \mathrm{~cm}$ de la pared. Todas las paredes internas, los cielorrasos y los aleros hasta $3 \mathrm{~m}$ de altura fueron tratados. Así también, la parte inferior y posterior de los muebles. En el peridomicilio se trataron gallineros y otros abrigos de animales, palos, tejas y las partes de los muros de piedra cercanos a la habitación.

Los potes fumígenos (PF5), producidos por los laboratorios Aguvac de Argentina, contenían bajas concentraciones de cypermetrina y DDVP $^{15}$. Ellos fueron utilizados en número de 2 por cuarto, una vez al mes, durante 3 meses, tratándose siempre de cerrar las aberturas de la habitación antes de encenderlos para que los humos no se perdieran. En el peridomicilio se aplicó insecticida convencional, en la dosis recomendada por el fabricante, también con la utilización de bombas dorsales, manuales, al igual que en el grupo control.

El grupo control recibió un único tratamiento convencional en cada país, o sea, propoxur en Chile ( $1 \mathrm{~g}$ de ingrediente activo $/ \mathrm{m}^{2}$ ) y deltametrina en Honduras y Paraguay ( $25 \mathrm{mg}$ de ingrediente activo $/ \mathrm{m}^{2}$ ), las dosis recomendadas por los fabricantes, también con la utilización de bombas dorsales, manuales y boquillas Teejet 8002 .

Se constituyeron cuatro grupos experimentales en cada uno de los países, incluyendo el grupo control que recibió rociado de insecticida convencional en el domicilio y en el peridomicilio. Uno de los otros grupos recibió 
Oliveira Filho AM. Uso de nuevas herramientas para el control de triatominos en diferentes situaciones entomológicas en el continiente americano. Revista da Sociedade Brasileira de Medicina Tropical 30:41-46, jan-fev, 1997.

pintura en el intra y peridomicilio; otro, pintura en el intra y rociado convencional en el peridomicilio, y el último, potes fumígenos dentro de la vivienda y rociado convencional en el peridomicilio. Los índices de infestación de las vivendas por triatominos previos a la intervención variaban de 14-28\% en Chile e de $20-40 \%$ en Honduras y Paraguay.

En cuanto al tamaño de la muestra, tres grupos de viviendas (unidad estadística) fueron conformados en localidades infestadas, seleccionadas al azar mediante números aleatorios. Cada grupo de estudio experimental se componía de 150 viviendas y el grupo control de 300. Para el seguimiento entomológico, el $50 \%$ de las viviendas fueron evaluadas por el método hora/hombre y el 50\% restante, con las cajas sensoras. Las evaluaciones fueron planeadas cada tres meses, a lo largo de dos años. Las viviendas en las que se detectó infestación después de la aplicación de las intervenciones serían tratadas con rociamiento tradicional intra y peridomiciliario y quedarían eliminadas del estudio ${ }^{8}$.

En Chile la especie vectora predominante era el $T$. infestans, pero también se registró la presencia de T. spinolai en los muros de piedra alrededor de las viviendas. Éstos no fueron totalmente tratados por la poca o ninguna importancia que tiene esta especie en la transmisión de la enfermedad de Chagas al hombre, pues en situación real no se alimentan de sangre humana ${ }^{13}$. En Paraguay predominaba también el T. infestans, mientras que en Honduras el $R$. prolixus y $T$. dimidiata.

\section{RESULTADOS}

La Tabla 1 se refiere a los porcentajes de viviendas reinfestadas seis meses después del inicio de las intervenciones con insecticidas en los cuatro grupos experimentales (a excepción de Chile donde no se trató con los potes fumígenos). En todos los países estudiados, los mejores resultados fueron observados en los grupos tratados con pintura en el intra y en el peridomicilio, donde se observó las más bajas tasas de reinfestación de las viviendas. Las más altas tasas de reinfestación se observaron en los grupos tratados con los potes fumígenos dentro del domicilio y rociamiento convencional en el peridomicilio que superaron siempre los números del control tratado con rociado convencional en el intra y peridomicilio.

Tabla 1 - Porcentajes de viviendas infestadas con triatominos seis meses después del tratamiento en el intra y peridomicilio con pinturas conteniendo el 8,3\% de Malathion, potes fumígenos conteniendo cypermetrina y DDVP o rociado convencional con propoxur (en Chile) o deltametrina (en Honduras y Paraguay).

\begin{tabular}{|c|c|c|c|c|c|}
\hline \multirow{2}{*}{$\begin{array}{c}\text { Grupos } \\
\text { experimentales }\end{array}$} & \multirow{2}{*}{$\begin{array}{c}\text { Tratamientos } \\
\text { intra/peri }\end{array}$} & \multirow{2}{*}{$\begin{array}{c}\text { № de } \\
\text { viviendas }\end{array}$} & \multicolumn{3}{|c|}{$\%$ Viviendas infestadas } \\
\hline & & & Chile & Honduras & Paraguay \\
\hline I & $\begin{array}{c}\text { pintura/ } \\
\text { convencional }\end{array}$ & 150 & $3,0^{*}$ & $8,0^{*}$ & 4,6 \\
\hline II & $\begin{array}{c}\text { potes/ } \\
\text { convencional }\end{array}$ & 150 & No det. & 18,6 & 8,0 \\
\hline III & $\begin{array}{l}\text { pintura/ } \\
\text { pintura }\end{array}$ & 150 & $0,0^{*}$ & $4,0^{*}$ & $2,6^{*}$ \\
\hline IV & $\begin{array}{l}\text { convencional/ } \\
\text { convencional }\end{array}$ & 300 & 4,5 & 11,0 & 3,3 \\
\hline
\end{tabular}

${ }^{*}$ Diferencias con el grupo control estadísticamente significativas ${ }^{10}$.

La Figura 1 nos muestra los datos de Chile hasta dos años después del tratamiento, desafortunadamente sin el grupo potes/convencional. Los resultados indican total control durante todo el periodo para el grupo de viviendas tratadas con pintura dentro y fuera, con solo $1 \%$ de las vivendas reinfestadas a los dos años. El peor nivel de control se obtuvo con tratamiento tradicional en el intra y peridomicilio, con 9\% de reinfestación a los años y una clara tendencia al aumento del número de viviendas reinfestadas.
De Chile también vienen los únicos resultados, que permiten una comparación preliminar entre los métodos de evaluación entomológica, o sea, la hora/hombre y las cajas sensoras. Hay que notar que en Chile fueron utilizados los "Sensores Serena", hechos localmente, muy parecidos a los "Sensores María”, hechos en Argentina, ambos derivados de la caja de Gómez-Nuñez, originária de Venezuela ${ }^{2}$. De las 24 casas positivas encontradas en los tres grupos observados en el periodo de un año, 14 (58,3\%) fueron 
Oliveira Filho AM. Uso de nuevas herramientas para el control de triatominos en diferentes situaciones entomológicas en el continiente americano. Revista da Sociedade Brasileira de Medicina Tropical 30:41-46, jan-fev, 1997.

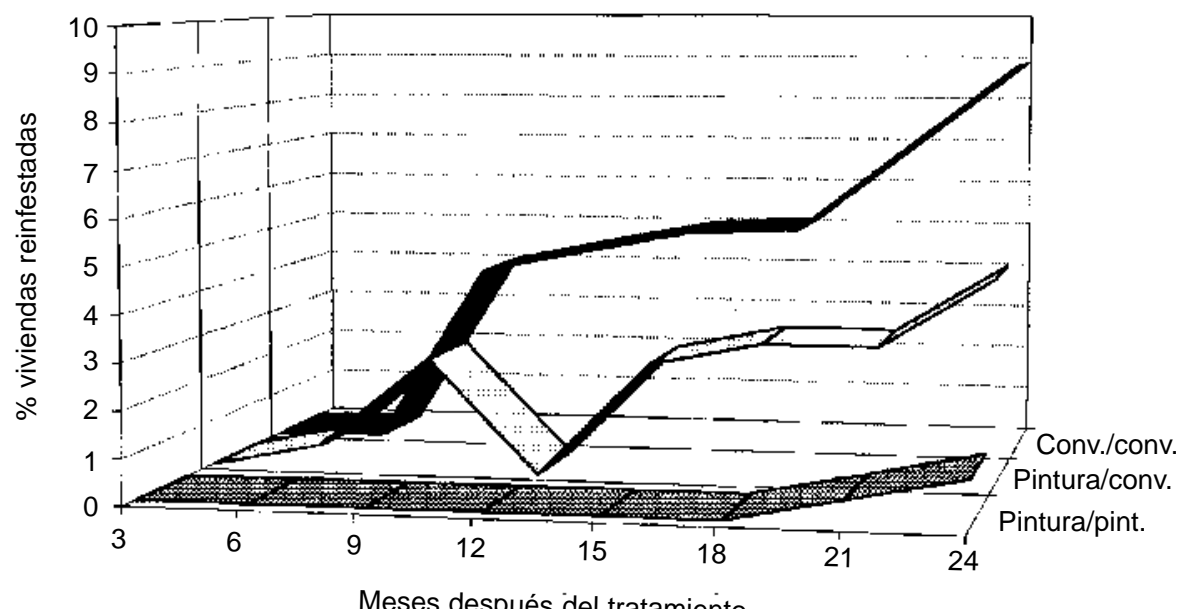

Meses después del tratamiento

Pintura/pint. $\square$ Pintura/conv. Conv./conv.

Figura 1 - Porcentajes de viviendas infestadas con T. infestans en el intra y en el peridomicilio después de un año de los tratamientos insecticidas realizados en Chile, con evaluaciones cada trimestre en el periodo de junio 91 a mayo $93^{1011}$.

identificadas como positivas por la técnica hora/hombre y $10(41,7 \%)$ por las cajas sensoras pasivas y permanentes, indicando preliminarmente mayor sensibilidad de la búsqueda por hombres entrenados, lo que confirma los resultados obtenidos anteriormente en Brasil ${ }^{12}$.

\section{DISCUSIÓN}

Las pinturas aplicadas en el intra y peridomicilio vienen demostrando una performance excelente, manteniendo los más bajos niveles de infestación hasta el momento, en todos los países observados. Esto tiene validez en los países con preponderancia de $T$. infestans (Chile y Paraguay), que se abriga más frecuentemente en las paredes de la casa, como también para el $R$. prolixus, que se abriga principalmente en el techo y que es preponderante en largas áreas de Honduras. Para el $T$. dimidiata, que también se presenta en Honduras y que se encuentra preferencialmente en el suelo suelto del interior de las viviendas, el grado de control fue más bajo, pero esto sucedió en todos los grupos de tratamiento.

Los potes fumígenos han demostrado baja eficacia hasta el momento pues esta herramienta no fue diseñada para tener poder residual. Además de esto, cuando utilizadas en fase de ataque para combatir grandes infestaciones triatomínicas, los potes tendrían que ser aplicados repetidamente (una vez cada mes, durante 3 meses) exigiendo altos costos operacionales? La necesidad de tratar el peridomicilio con otro insecticida de acción residual complicaría todavía más su empleo en estas circunstancias. Quizás su utilización se adapte mejor a la vigilancia echa por la propia población en un programa de participación popular. En este sentido se podría plantear que los potes pueden ser uma técnica complementaria a las pinturas o tratamientos convencionales, pudiendo quizás ser utilizados durante el período de vigilancia después del tratamento inicial con insecticida de acción residual.

Los datos de Chile indican mayor sensibilidad de la técnica hora/hombre que la de las cajas sensoras para detectar infestaciones por $T$. infestans. La hora/hombre hace un corte momentáneo en la población, indicando lo que pasa en aquel momento, de suerte que es más apropiada para hacerse investigaciones sobre formulaciones y técnicas de control. Las cajas sensoras ${ }^{13}$, así como simples hojas de papel pegadas a las paredes cercanas de los sitios donde duermen hombres o animales 
Oliveira Filho AM. Uso de nuevas herramientas para el control de triatominos en diferentes situaciones entomológicas en el continiente americano. Revista da Sociedade Brasileira de Medicina Tropical 30:41-46, jan-fev, 1997.

(utilizadas en algunas ocasiones en Brasil y Paraguay), pueden servir para el monitoreo en casos de bajas densidad triatomínica, durante periodos de vigilancia hechos después de un tratamiento residual donde no se exige mucha precisión de datos, sino solamente indicaciones para promover el retratamiento en los domicilios infestados. Esta técnica tiene todavía la desventaja de sólo monitorear el interior de la vivienda, donde se encuentran protegidas del sol y de la lluvia, dejando sin observar el peridomicilio donde suelen aparecer más frecuentemente las infestaciones.

Con relación a las pinturas insecticidas de lenta liberación, algunas dificultades operacionales fueron superadas en la experiencia de entrenamiento de los rociadores en los países participantes. Las obstrucciones suelen ser evitadas si se sacan todos los filtros de las bombas, los cuales son desnecesarios pues esta formulación es muy homogénea y no presenta gránulos. Se tiene noticia de algunas quejas de parte de los rociadores en cuanto al olor del organofosforado, aunque esta formulación ya lo haya atenuado. También se quejaron de tener que limpiar muy bien su equipo para evitar la formación de películas en el interior de la bomba cuando trasnochaba ahí la formulación. Sin embargo, se solucionaron tales problemas a medida que los rociadores se adaptaron al nuevo producto, lo que ocurrió después de su utilización durante algún tiempo.

\section{SUMMARY}

The field results of new tools for triatomine control developed under the sponsorship of WHO/TDR are reported: a) A slow-release "paint" containing malathion; b) fumigant cans containing cypermethrin and DDVP, and c) sensor boxes for the detection of triatomine infestations. Field assays were performed in Chile, Honduras and Paraguay against Triatoma infestans, T. dimidiata and Rhodnius prolixus, accordingly to a standard protocol designed by a WHO experts committee. Preliminary 6 months post-treatment results for the three countries show an efficient control when insecticide paints were used indoors and in the peridomicilium, keeping reinfestation near zero. The final results presented for Chile two years post-treatment confirmed the superiority of the slow-release "paints". Sensor boxes were less effective than man/hour captures in the detection of infested houses.

Key-words: Insecticides. Slow-release formulation. Triatomines. Chagas' disease.

\section{AGRADECIMIENTOS}

Mi gratitud al Programa COROLUCHA de Argentina, OMS y OPS por la invitación para presentar este trabajo en el "Taller de Educación y Vivienda en la Promoción de la Salud", Resistencia, Argentina del 31/03 al 03/04/93. Agradezco a todos los participantes de los proyectos de la OMS en Chile, Paraguay y Honduras (especialmente a D. Julio Valdés y al Dr. Víctor Correa, de Chile; a los Dres. Carlos y Elisabeth Ponce, de Honduras y la Dra. Sofia Cardozo, de Paraguay) por los datos enviados en sus relatos al TDR. Agradezco también al personal del Laboratorio de BiologíaNPPN/UFRJ, Marli Melo, Celso Santos, Elza Lustosa, Servaas Engels, Orbino Damião, Joaquim Silva, Elizabete Costa y Aline Oliveira, por su constante colaboración. Por la revisión del texto agradezco a Rosa Maria da Silva-UNESP y al padre Felipe Conde Pardo. Apoyo WHO/UNDP-World Bank/TDR, CNPq, CEPEGUFRJ.

\section{REFERÊNCIAS BIBLIOGRÁFICAS}

1. Apt W, Aguilera X, Arribada A, Gomez L, Miles MA, Widmer G. Epidemiology of Chagas disease in northern Chile: isozyme profiles of Trypanosoma cruzi from domestic and silvatic transmission cycles and their association with cardiopathy. The American Journal of Tropical Medicine and Hygiene 37:302-307, 1987.

2. Gómez-Núñez JC. Desarrollo de um nuevo método para evaluar la infestación intradomiciliaria por Rhodnius prolixus. Acta Cientifica Venezolana 16:26-31, 1965.

3. Miles MA. Triatomine vectors and Trypanosoma cruzi zymodemes in Chile. In: Taller sobre erradicación o control de la enfermedad de Chagas en Chile. Sociedad Chilena Parasitologia y Colegio de Chile, Santiago, p. 9, 1991.

4. Oliveira Filho AM. New Alternatives for Chagas' disease control. Memórias do Instituto Oswaldo Cruz 79 (supl):117-123, 1984.

5. Oliveira Filho AM. Development of insecticide formulations and determination of dosages and application schedules to fit specific situations. Revista Argentina de Microbiologia 20 (supl):3948, 1988.

6. Oliveira Filho AM. New alternatives for the control of triatomines in peridomestic buildings. Revista da Sociedade Brasileira de Medicina Tropical 22 (supl .II):53-57, 1989. 
Oliveira Filho AM. Uso de nuevas herramientas para el control de triatominos en diferentes situaciones entomológicas en el continiente americano. Revista da Sociedade Brasileira de Medicina Tropical 30:41-46, jan-fev, 1997.

7. Oliveira Filho AM. Cost-effectiveness analysis in Chagas' disease vectors control interventions. Memórias do Instituto Oswaldo Cruz 84 (supl IV):409.417, 1989a.

8. Organización Mundiale de la Salud. Protocolo estandar para el ensayo de nuevas estrategias de control de vectores de la enfermedad de Chagas. Doc.TDR/CHA/URU/89.3, 1989.

9. Organización Mundiale de la Salud. Control of Chagas Disease. WHO,Technical Report Series 811:47-51, 1991.

10. OMS. Progress reports of principal investigators of projets in Chile, Honduras and Paraguay, in charge of the assay on new tools for the control of Chagas' disease vectors,TDR - Geneva, 1993.

11. Organización Mundiale de la Salud. Paints make houses hostile to Chagas' bug in Chile. TDR News, 43:13, 1993.
12. Pinchin R, Fanara DM, Castleton CW, Oliveira Filho AM.A comparative study of domestic survey techniques for the Chagas' disease vector Triatoma infestans. Insect Science and Applications 3:7984, 1982.

13. Segura EL. Sensor María una propuesta para la evaluación entomológica pasiva intradomiciliaria de triatominos. In: Taller de Educación y vivienda en la promoción de la salud, Resistencia, Argentina, 1993.

14. Vlassoff C. Listening to the people-improving disease control using social sciences approaches. Transactions of the Royal Society of Tropical Medicine and Hygiene 86:465-466, 1992.

15. Zerba E. Pote fumígeno insecticida. In: Taller de Educación y vivienda en la promoción de la salud, Resistencia, Argentina, 1993. 